\title{
DELIRIUM SCREENING IN ACUTE STROKE A SYSTEMATIC REVIEW AND META-ANALYSIS OF INCIDENCE, SCREENING TOOLS AND SCREENING FREQUENCIES
}

\section{J. Stokholm ${ }^{1}$, J. Steenholt ${ }^{1}$, T. Kjær ${ }^{2}$, T. Christensen ${ }^{1}$.}

1Nordsjællands Hospital - Hillerød, Department of Neurology, Hillerød, Denmark.

2Zealand University Hospital- Roskilde, Department of Neurology - Center for Neurophysiology, Roskilde, Denmark.

\section{Introduction}

Delirium in acute stroke patients is a serious complication with adverse effects on functional outcome. The incidence of delirium during the hospitalization for acute stroke varies across studies.

We performed a systematic literature review to address whether the choice of delirium screening tool, frequency of use and the type of health professional carrying out the screening influence the number of delirious patients detected in a population of acute stroke patients.
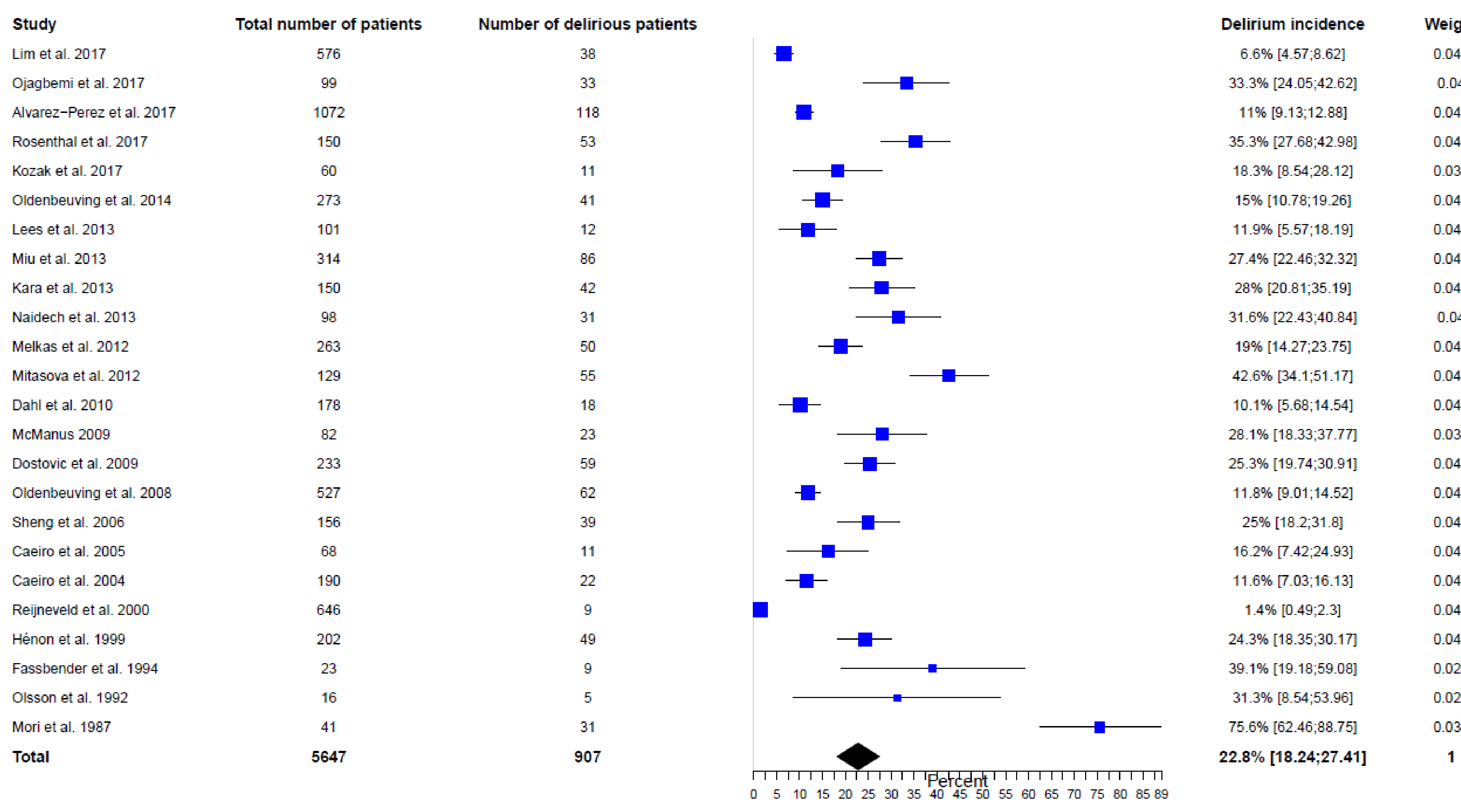

Forest plot. The size of each square visualizes a study's percentile weight, the horizontal bars indicate the $95 \% \mathrm{Cl}$ The center of the diamond indicates the overall delirium incidence estimate and the width of the diamond represents the $95 \% \mathrm{Cl}$ of the overall estimate.

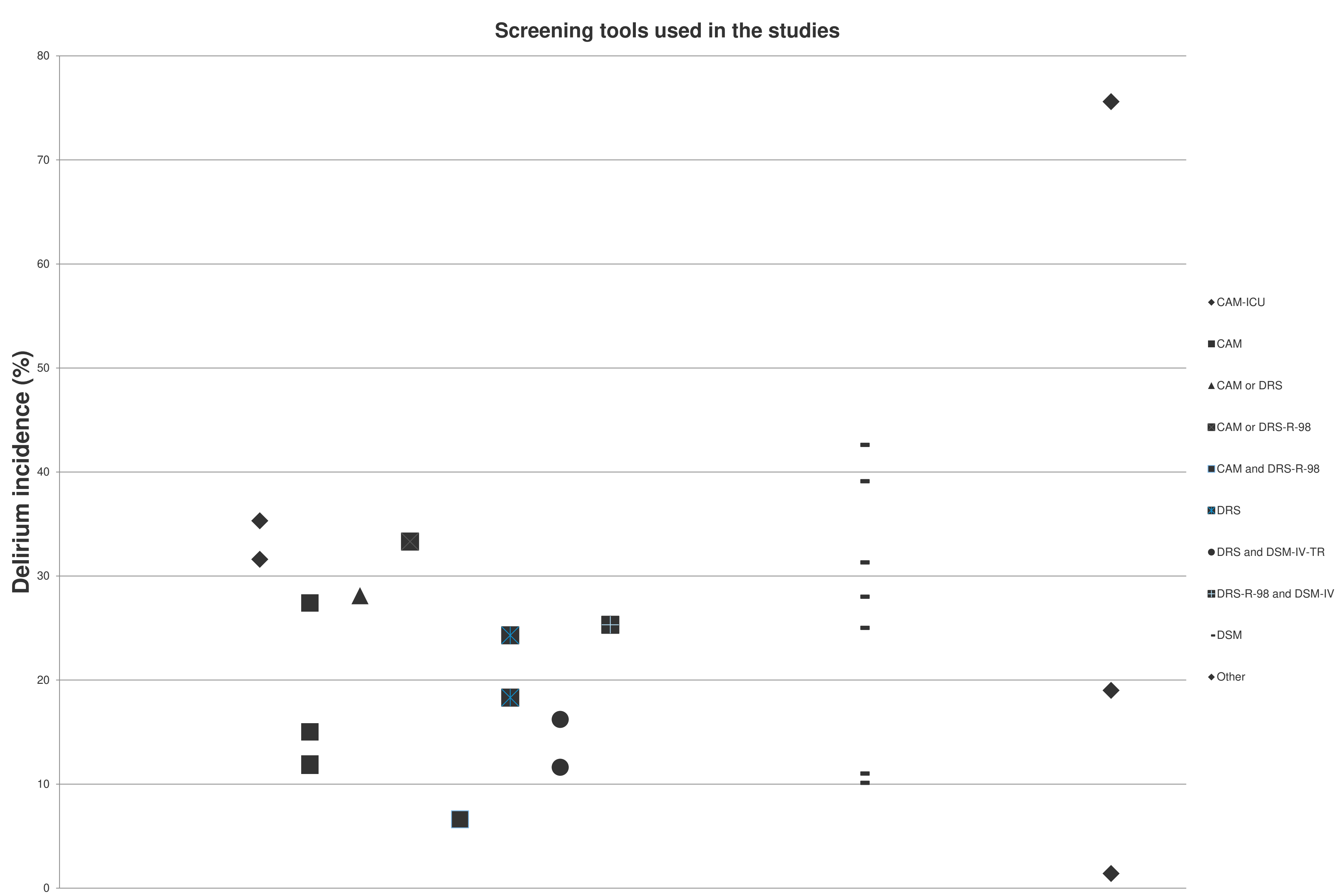

\section{Methods}

A systematic review protocol was registered at the PROSPERO register. We searched MEDLINE, EMBASE and PsycINFO and included pro- and retrospective cohort studies which tested for delirium in any way during hospitalization of adult patients with acute stroke. Article screening, selection and data extraction were carried out independently by two reviewers.

\begin{abstract}
Results
24 unique populations were included in the review from 22 prospective studies and 2 retrospective studies. Delirium incidence ranged from $1.4 \%$ to $75.6 \%$ in total and narrowed down to approx. $10 \%$ to $30 \%$ for studies using the screening tools Confusion Assessment Method (CAM) and Delirium Rating Scale (DRS(-R-98)). There was no clear correlation between delirium incidence and the frequency of delirium screening or the type of health professional using a given tool.
\end{abstract}

Concerning screening frequencies, 6 prospective studies screened once a day or more and the remaining prospective studies screened with a smaller frequency, e.g. twice a week. The retrospective studies used records from either the entire admission or were limited to days 1 through 7 .

\section{Discussion}

The broad range in delirium incidence reported may to some extent be explained by the choice of screening tool, but the different tools may also underor over-diagnose some delirium subtypes.

We found no discernable pattern in terms of delirium incidence and screening frequency or screening too and screening frequency. However, there were too many different tools in use across the 22 prospective cohorts for a conclusion to be made with respect to a possible association between the screening frequency and delirium incidence for any of the tools.

Delirium is a common complication with a heterogeneous presentation of symptoms that can be difficult to recognize in some patients and may be wrongly diagnosed in others. 